\title{
A longslit spectroscopic survey of bulges in disc galaxies
}

\author{
Maximilian Fabricius ${ }^{1}$, Roberto Saglia ${ }^{1}$, David Fisher ${ }^{2}$, Niv Drory $^{3}$, \\ Ralf Bender ${ }^{1}$ and Ulrich Hopp ${ }^{1}$ \\ ${ }^{1}$ Max Planck Institute for Extraterrestrial Physics \& University Observatory, Munich, \\ Germany, email: mxhf@mpe.mpg.de \\ ${ }^{2}$ Laboratory of Millimeter Astronomy, University of Maryland \\ ${ }^{3}$ Instituto de Astronomia, Universidad Nacional Autonoma de Mexico (UNAM)
}

\begin{abstract}
We use the Marcario Low Resolution Spectrograph (LRS) at the Hobby-EberlyTelescope (HET) to study the kinematics of pseudobulges and classical bulges in 45 S0-Sc type galaxies in the nearby universe. Our high-resolution (instrumental $\sigma \approx 39 \mathrm{~km} \mathrm{~s}^{-1}$ ) spectra allo only to resolve the typical velocity dispersions of our targets but also to derive the h3 and h4 Gauss-Hermite moments. We demonstrate for the first time that purely kinematic diagnostics of the bulge dichotomy agree systematically with those based on Sérsic index. Low Sérsic index bulges have both increased rotational support (higher $\mathrm{v} / \sigma$ values) and on average lower central velocity dispersions. Pseudobulges have systematically shallower velocity dispersion profiles. The same correlation also holds when visual morphologies are used to diagnose bulge type. Finally, we present evidence for formerly undetected counter rotation in the two systems NGC 3945 and NGC 4736 . With these, a total of $16 \%$ of the systems in or sample show signs for stellar counter rotation.
\end{abstract}

